\title{
The Different Impact of a Half-Separated Gravel and Vegetated Bed in Open Channels
}

\author{
E. Keramaris $^{1}$ - G. I. Pechlivanidis ${ }^{2}$ - I. G. Pechlivanidis ${ }^{3}$
}

Received: 16 December 2014 / Accepted: 20 July 2015 /Published online: 27 August 2015

(C) Springer International Publishing Switzerland 2015

\begin{abstract}
This paper investigates the different impact of a half-separated gravel and vegetated bed in open channels. This experimental investigation is based on the 2D Particle Image Velocimetry (PIV) method, which allows data acquisition at fine spatial-temporal resolution. For the simulation of the two porous beds : a) a grasslike vegetation and b) gravel bed of $2 \mathrm{~cm}$ height were used to represent the permeable bed. Velocity is measured : a) at the central line of $3.75 \mathrm{~cm}$ above the interface of vegetated and gravel bed, b) over the vegetated bed and c) over the gravel bed for different discharges and for the same different total heights. In total, 27 experiments were carried out. Results show that the presence of a half-separated gravel and vegetated bed influences significantly the velocity distribution in comparison with a simple permeable bed (vegetated or gravel). This is due to the fact that the presence of gravel bed in the half-separated bed decreases the velocity of the flow in regard to the simple vegetated or gravel bed due to the great roughness which is observed near the surface gravel-vegetated bed. This fact increases the turbulence and reduces the velocity. Also, the presence of half-separated gravel and vegetated bed influences the values of velocity distribution in a different way in comparison with situations over of half-separated impermeable and permeable bed.
\end{abstract}

Keywords Half-separated bed $\cdot$ Permeable bed $\cdot$ PIV $\cdot$ Turbulent flow $\cdot$ Experimental analysis

\section{E. Keramaris}

ekeramaris@civ.uth.gr

1 Department of Civil Engineering, University of Thessaly, Volos 38334, Greece

2 Department of Civil Infrastructure Engineering, Alexander Technological Educational Institute of Thessaloniki, Sindos 57400 Thessaloniki, Greece

3 Swedish Meteorological and Hydrological Institute, Folkborgsvägen 1, SE - 60176 Norrköping, Sweden 


\section{Introduction}

The effects on the velocity distribution of turbulent flow in an open channel with a halfseparated impermeable and permeable bed is of particular importance. In nature, a growth of vegetation is observed both in rivers and open channels. The presence of a permeable bed in an open channel has as a result the change of velocity distribution. The changes are greater when this permeable bed is half-separated gravel and vegetated bed.

The effects of porous bed, for laminar flow with low-Reynolds numbers near the porous/ fluids interface or porous/air interface, have been studied computationally, analytically and experimentally by many investigators (e.g., Beavers and Joseph 1967; Poulikakos and Kazmierczak 1987; Vafai and Thiyagaraja 1987; Sahraoui and Kaviany 1992; Choi and Waller 1997).

The study of turbulent flow over and within a permeable layer is rather limited since there are further difficulties because of turbulence. The first complete study for the turbulent flow has been realized by Mendoza and Zhou (1992). They found analytical results for the turbulent flow characteristics over a porous bed and for the velocity distribution within the porous bed.

In the study of Velasco et al. (2003), experimental tests were conducted under controlled conditions. In order to simplify the inherent complexity of vegetation properties, scaled plastic strips were used to model plants. Different plant densities were tested as well as different flow conditions such as the flow and the ratio of the plant height to the water depth. The velocity field was measured directly in the experimental flume with an Acoustic-Doppler sensor (ADV). The results from the velocity measurements show a reduction in friction factors due to plant bending for an increasing Reynolds number as expected.

Wilson et al. (2003) carried out laboratory experiments to study the effect of flexible vegetation on open channel flow. They used two different forms of vegetation: a) flexible rods of constant height; and b) the same rods with a front foliage attached. Experimental results revealed that within the plant layer, the velocity profile no longer followed the logarithmic law profile and the mean velocity for the rod/frond canopy was less than half of that observed for the simple rod array.

Chan et al. (2007) examined numerically the characteristics of fully developed turbulent flow in a hybrid domain channel, which consists of a clear fluid region and a porous bed, using a model based on the macroscopic Reynolds-averaged Navier-Stokes equations. The effects of porosity and Darcy number on the flow properties over and inside the porous bed are further investigated. The thickness of turbulent penetration remains proportional to the values of porosity and Darcy number. Moreover, turbulent shear stress within the upper part of porous bed increases significantly with the porosity and Darcy number.

Bouma et al. (2007) combined field, flume and numerical experiments to identify spatial sedimentation and erosion patterns developed within rigid vegetation patches and assessed the relevance of hydrodynamic flume studies for the longterm sediment dynamics in the field. Ghisalberti and Nepf (2009) investigated experimentally the distinguishing hydrodynamic features of flows with rigid and semi-rigid vegetation patches and found that the length at which the flow was fully-developed was governed by the growth of the vortex street and the mixing-layer.

Huai et al. (2009) used a PVC slice to simulate flexible vegetation and carried out experiments in an open channel with submerged flexible vegetation. A 3D acoustic Doppler velocimeter (micro ADV) was used to measure local flow velocities and Reynolds stress. The results show that hydraulic characteristics in non-vegetation and vegetation layers are totally 
different. In a region above the vegetation, the Reynolds stress distribution is linear and the measured velocity profile is a classical logarithmic one.

Keramaris et al. (2013) carried out experiments to investigate the impact of lateral walls on the velocity profile in an open channel. Although the width of the channel was only $7.5 \mathrm{~cm}$, it did not influence the magnitude of the velocities. Results from these experiments showed that the lateral walls influenced the velocities at a distance limited to $0.4 \mathrm{~cm}$ from the walls. This result indicates that the wall does not influence the instantaneous velocities in the central area of the channel where the velocity measurements are usually conducted. The impact of the lateral walls on the flow dynamics in the rest of the channel is negligible.

A series of laboratory experiments were performed by Zhu et al. (2014) to investigate the connection between submerged vegetation and the key geomorphology around the vegetation. The submerged vegetation was modeled as bundled plastic fibers with a variation in vegetation density. The objective of this work was to describe the detailed characteristics of flow through a simulated array of rigid vegetation by examining its effects on the velocity, and observing the influence of vegetation density and heights. Measurements of velocity were taken along verticals at multiple locations at different sections to adequately represent the conditions everywhere within the flow and to capture the flow response as it moves through the vegetation array.

Finally Keramaris and Pechlivanilis (2015) studied experimentally, using a 2-D Particle Image Velocimetry (PIV), the effects on the velocity distribution of turbulent flow in an open channel in a half-separated (impermeable and permeable) bed. A grass-like vegetation of $2 \mathrm{~cm}$ height was used for the simulation of the half permeable bed (with $3.75 \mathrm{~cm}$ width). Velocity was measured at the centreline $3.75 \mathrm{~cm}$ above the vegetation for the permeable bed at the corresponding point above the impermeable bed as well as at the interface between impermeable and permeable bed, in the mid-plane of the channel. Results showed that the presence of half-separated impermeable and permeable bed influences the values of velocity distribution in comparison with situations over permeable or impermeable bed. The comparison with the same experiments when it has transition from permeable to impermeable bed and vice versa shows that there are a lot of differences on velocity distribution.

In this study, the different impact of a half-separated gravel and vegetated bed in open channels is investigated experimentally. Results show that the presence of a half-separated gravel and vegetated bed influences significantly the velocity distribution in comparison with a simple permeable bed (vegetated or gravel) or over a half-separated impermeable and permeable bed.

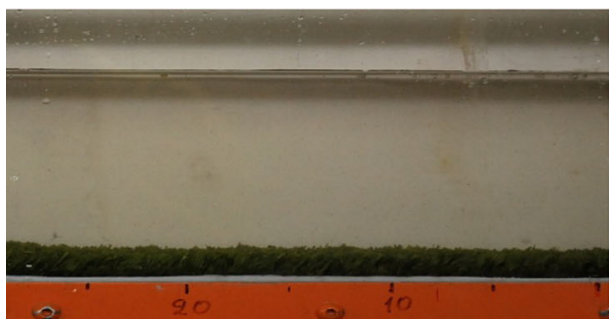

a)

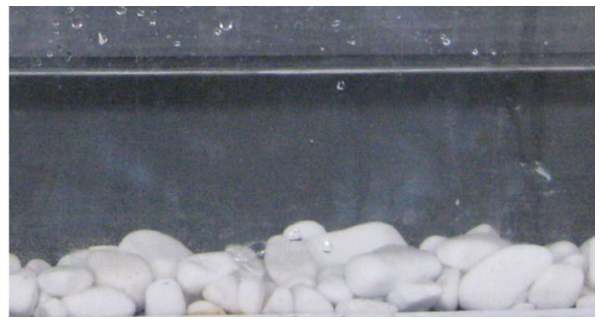

b)

Fig. 1 a Grass vegetation of $2 \mathrm{~cm}$ height; $\mathbf{b}$ Gravel bed of $2 \mathrm{~cm}$ height 


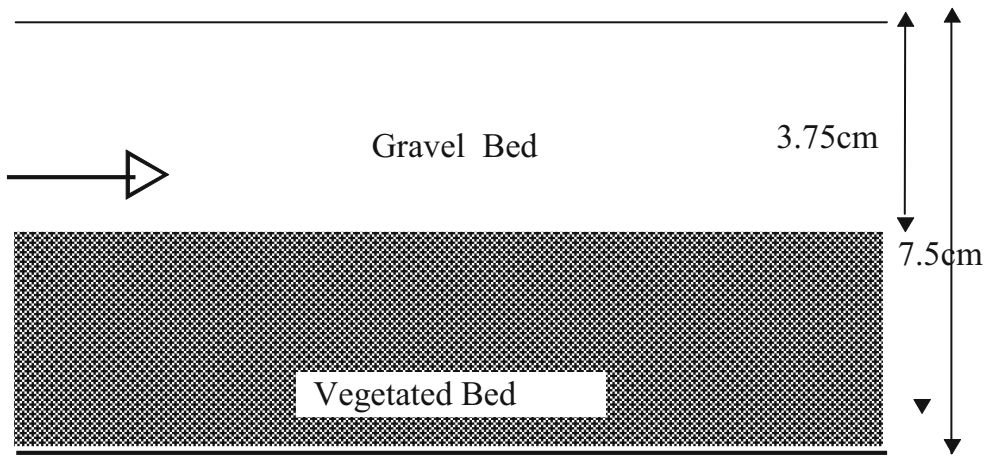

Fig. 2 Plan view of the bottom of the channel

\section{Experimental Apparatus - Measurements}

In total, 27 experiments were carried out in the Laboratory of Hydraulics in the Department of Civil Infrastructure Engineering of Alexander Technological Educational Institute of Thessaloniki, Greece. The experiments were conducted in a horizontal channel, $6.5 \mathrm{~m}$ long, $7.5 \mathrm{~cm}$ wide and $25 \mathrm{~cm}$ deep. For the simulation of the two porous beds: a) a grass-like vegetation; and b) a gravel bed of $2 \mathrm{~cm}$ height were used to represent the permeable bed. Velocity was measured: a) at the centerline at $3.75 \mathrm{~cm}$ above the permeable bed; b) over the vegetated bed; and c) over the gravel bed for three different discharges $(\mathrm{Q}=0.735,0.845$ and $0.970 \mathrm{~L} / \mathrm{s}$ ) and for the same flow depths. The flow depths were $h=4.5,7$ and $10 \mathrm{~cm}$. The length

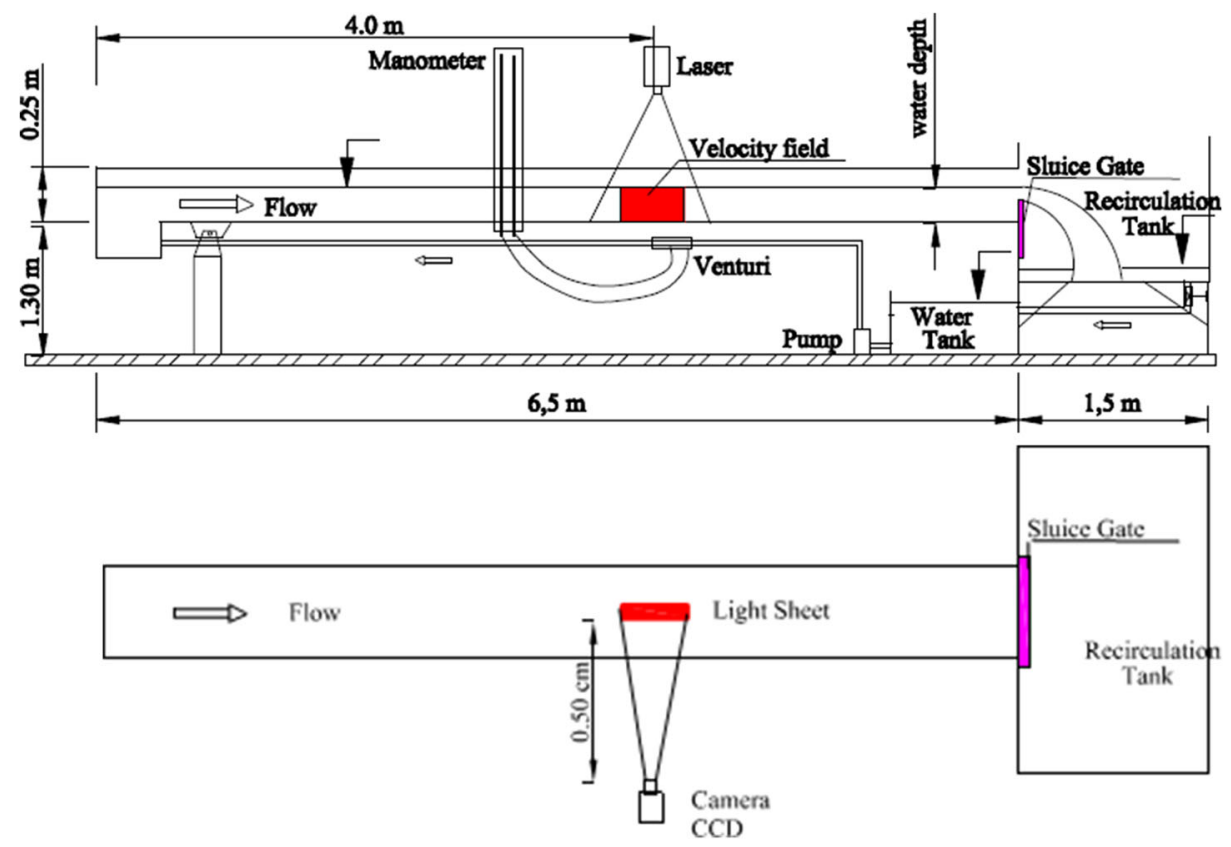

Fig. 3 Experimental set-up 


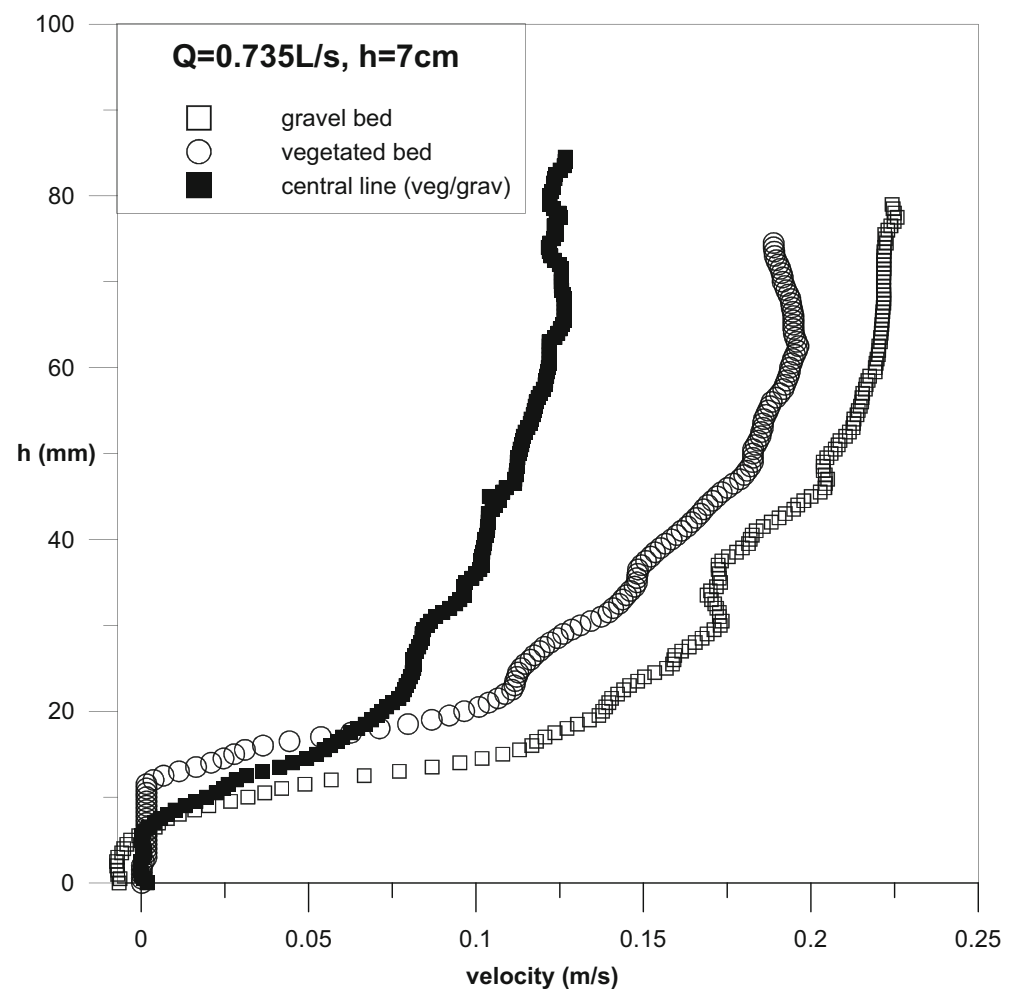

Fig. 4 Velocity profiles for the same discharge $\mathrm{Q}=0.735 \mathrm{~L} / \mathrm{s}$ and flow depth $h=7 \mathrm{~cm}$

of the two porous beds was $6.0 \mathrm{~m}$. The porous bed began $0.5 \mathrm{~m}$ after the channel entrance, so this was adequate for fully developed flow.

Measurements of the velocity were taken for the horizontal channel using a 2-D Particle Image Velocimetry (PIV). The PIV system used for the measurement of the velocity distribution in the flow domain consists of a twin pulsed Nd: Yag lasers (532 nm wavelength, 300 mjoule/pulse at $10 \mathrm{~Hz}$ ), a cross correlation 8 bit $1 \times 1 \mathrm{~K}$ CCD camera (Kodak MEGAPLUS ES 1.0), a synchronizer, a computer, an image acquisition system and a PIV analysis software (Insight 3G). The laser beams were combined and formed a 1-mm wide sheet by using semicylindrical optics. The camera image size creates a $1600 \times 1192$ pixel array and the dimension of the velocity field was kept to $120 \times 110 \mathrm{~mm}$ for all the experiments. The laser was installed above the open channel at a distance of $50 \mathrm{~cm}$ from the free water surface, while the camera viewed from an orthogonal direction (Fig. 2).

Twin images were recorded with a temporal interval of $1.5 \mathrm{~ms}$. In total, 200 pairs of images were captured per experiment. The plane photographs were divided into interrogation spots measuring $16 \times 32$ pixels $(1.2 \times 2.4 \mathrm{~mm})$. The fluid was generally seeded with tracer particles that, for the purposes of PIV, are generally assumed to follow the flow dynamics (Wereley and Meinhart 2010). These particles have a size of about $10 \mu \mathrm{m}$ in clean water. The motion of the seeding particles is used to calculate the velocity vectors of the flow. The distance between two neighbour velocity vectors is $1.5 \mathrm{~mm}$. From the velocity field, we can find the profile of the flow at each vertical direction or the mean space profile in the area. PIVs use the particle 


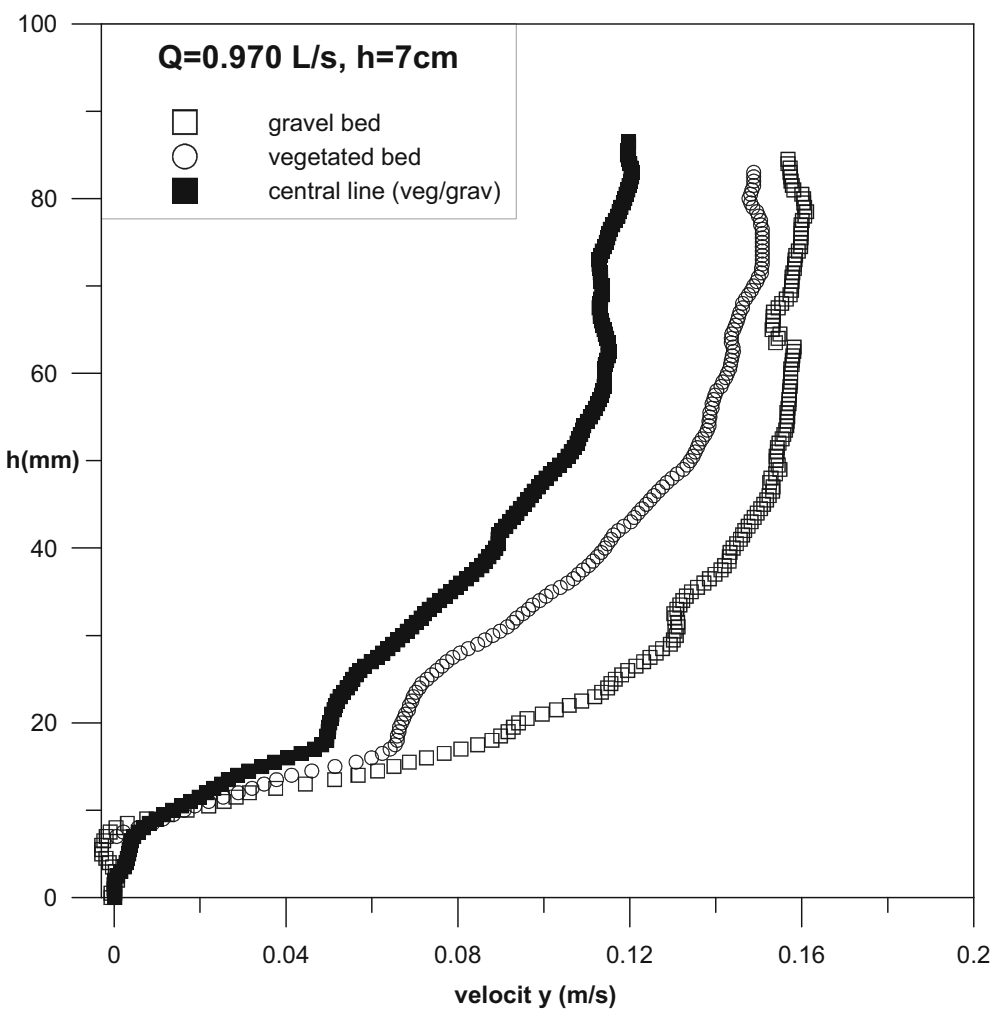

Fig. 5 Velocity profiles for the same discharge $\mathrm{Q}=0.970 \mathrm{~L} / \mathrm{s}$ and flow depth $h=7 \mathrm{~cm}$

concentration method to identify individual particles in an image and follow their flow (Raffel et al. 2007). The experimental uncertainty of the measured velocity with this technique is approximately $\pm 2 \%$.

The measurements were conducted at $4 \mathrm{~m}$ distance from the channel entrance, where the flow is considered fully developed. The full development of the flow was checked with the comparison of the velocity distributions in two vertical sections located $60 \mathrm{~cm}$ apart. Several point gauges for the check of the uniformity of the flow were used. A weir at the channel outlet controlled the desirable flow depth. The error of the measured flow depth $(10 \mathrm{~cm})$ with the point gauge was $\pm 0.1 \mathrm{~mm}$.

The morphology of the permeable bed (vegetated and gravel bed) is illustrated in Fig. 1. A plan view of the bottom of the channel is given in Fig. 2. The experimental apparatus is presented in Fig. 3 .

\section{Analysis of the Results}

Experiments were performed for the flow determination and for investigation of the different impact of a half-separated gravel and vegetated bed on the velocity distribution. In total, 200 pairs of images were captured per experiment. The validation of the images was based on a PIV analysis software (Insight 3G). From the velocity fields, the velocity profiles at various positions were determined using MatLab, which is integrated in the Insight $3 \mathrm{G}$ program. 

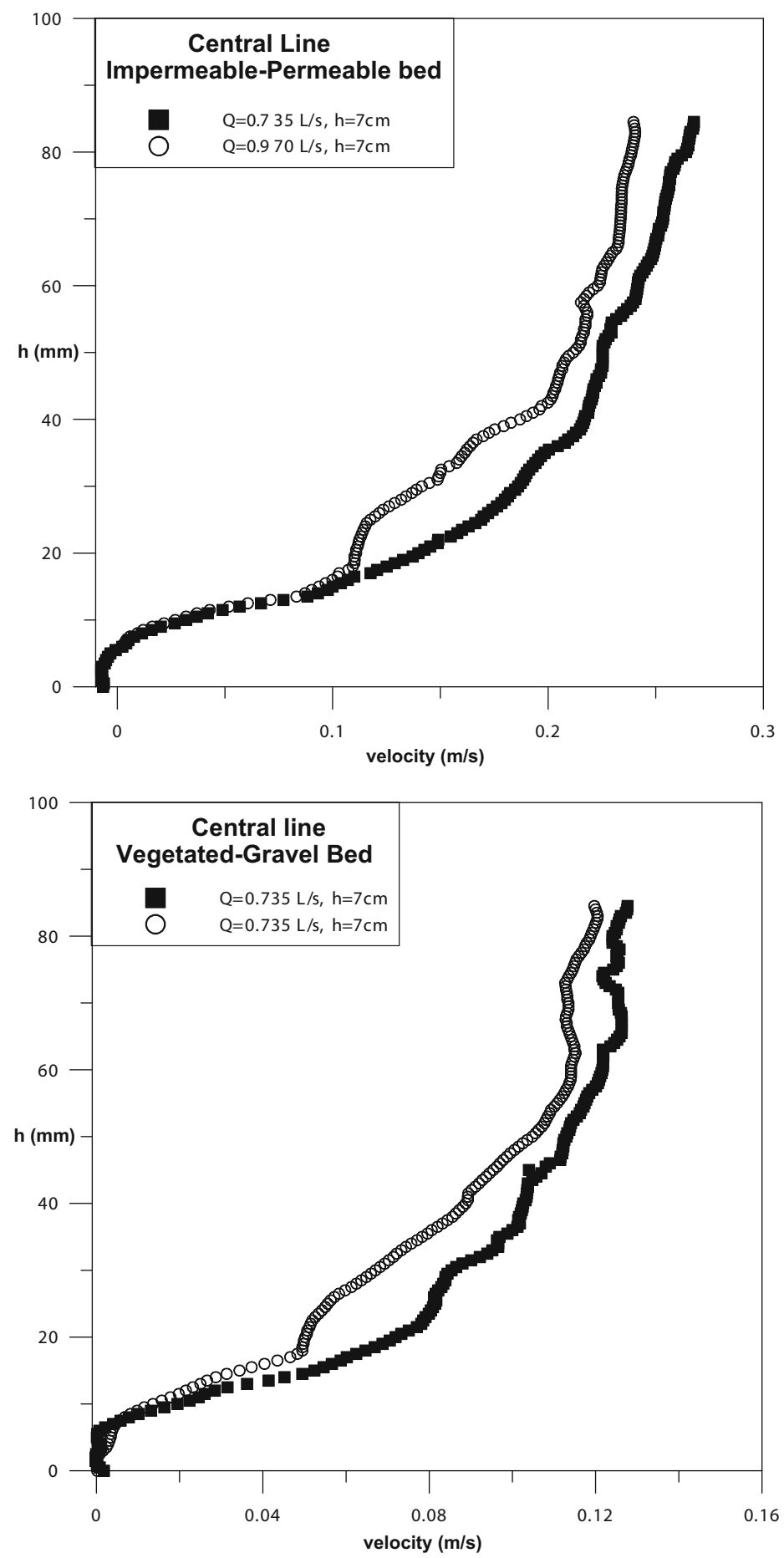

Fig. 6 Velocity profiles in central line for: a half-separated impermeable and permeable bed; and b halfseparated gravel and vegetated bed 


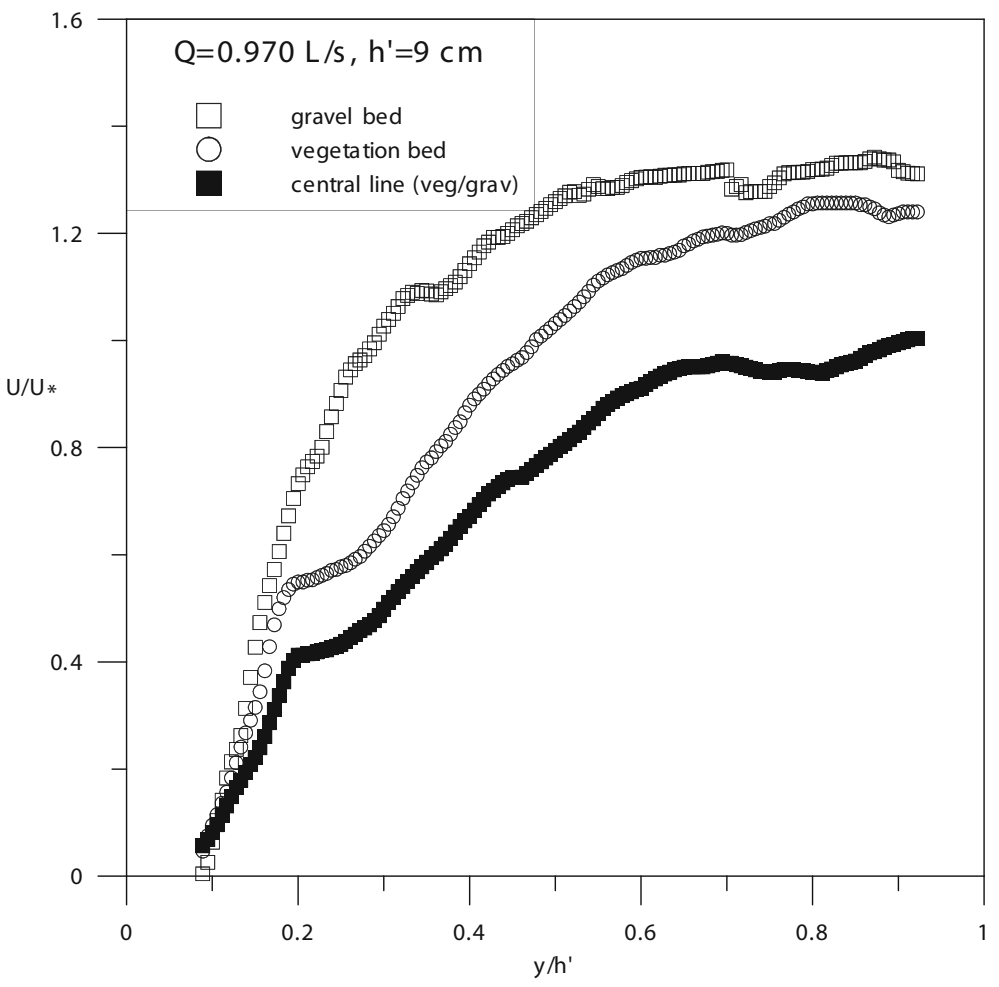

Fig. 7 Velocity profiles for the same discharge $\mathrm{Q}=0.970 \mathrm{~L} / \mathrm{s}$ and total flow depth $\mathrm{h}=9 \mathrm{~cm}$

Figures 4 and 5 present the profiles of the mean velocities for the three different discharges and for the three different positions: a) at the central line of $3.75 \mathrm{~cm}$ above the interface of vegetated-gravel bed; $b$ ) over the vegetated bed; and c) over the gravel bed and the same flow depth $\mathrm{h}=7 \mathrm{~cm}$ and for different discharges $(\mathrm{Q}=0.735$ and $\mathrm{Q}=0.970 \mathrm{~L} / \mathrm{s})$. As shown in these figures, the presence of a half-separated gravel and vegetated bed influences significantly the velocity distribution in comparison with a simple permeable bed (vegetated or gravel). This is due to the fact that the presence of gravel bed in the half-separated bed decreases the velocity of the flow in regard to the simple vegetated or gravel bed due to the great roughness which is observed near the surface gravel-vegetated bed. This fact increases the turbulence and reduces the mean velocity significantly, especially in the case of $Q=0.735 \mathrm{~L} / \mathrm{s}$.

Results show that at the central line of $3.75 \mathrm{~cm}$ the presence of half-separated gravel and vegetated bed influences the values of velocity distribution differently in comparison to situations over of half-separated impermeable and permeable bed. There are a lot of differences on velocity distribution with the same experiments (Keramaris et al. 2014) for half-separated impermeable and permeable bed. The mean velocities at the central line of $3.75 \mathrm{~cm}$ in Fig. $6 \mathrm{a}$ (half-separated impermeable and permeable bed) are much greater in comparison with those in Fig. 6b (half-separated gravel and vegetated bed). This is due to the fact that the high mixing and the development of the turbulence in the second case, because of the presence of gravel bed in the half-separated bed, decreases significantly the velocity of the flow in comparison with half-separated impermeable and permeable bed. 
In Fig. 7 the results are given in dimensionless form. The normalized streamwise mean velocity distribution $\left(\mathrm{U} / \mathrm{U}_{*}\right)$ is compared with the logarithmic law plotted versus the normalized depth $y / h$ ' (y: distance from bed, h': total flow depth). The differences in shear velocity of the flow above the different porous beds is important and explaining the above results. The difference in shear velocity above the different porous beds is the cause of a wake-type behavior of the flow at the central line of the channel.

\section{Conclusions}

The different impact of a half-separated gravel and vegetated bed in open channels is investigated experimentally. For the simulation of the two porous beds: a) a grass-like vegetation; and b) a gravel bed of $2 \mathrm{~cm}$ height were used to represent the permeable bed. The following conclusions can be derived:

- The main and significant conclusion of this study is that the presence of a half-separated gravel and vegetated bed influences significantly the velocity distribution in comparison with a simple permeable bed (vegetated or gravel). This is due to the fact that the presence of gravel bed in the half-separated bed decreases the velocity of the flow in regard to the simple vegetated or gravel bed due to the great roughness which is observed near the surface gravel-vegetated bed. This fact reduces the mean velocity. This conclusion is very clear in the case of dimensionless form in Fig. 7.

- The presence of half-separated gravel and vegetated bed influences the values of velocity distribution differently in comparison with situations over a half-separated impermeable and permeable bed. The mean velocities at the central line of $3.75 \mathrm{~cm}$ in the half-separated impermeable and permeable bed are greater in comparison with those in the half-separated gravel and vegetated bed. This is due to the fact that the high mixing and the development of the turbulence in the second case, because of the presence of gravel bed in the halfseparated bed, decreases significantly the velocity of the flow in comparison with halfseparated impermeable and permeable bed.

Acknowledgments This research has been co-financed by the European Union (European Social Fund - ESF) and Greek national funds through the Operational Program "Education and Lifelong Learning" of the National Strategic Reference Framework (NSRF) - Research Funding Program: ARCHIMEDES III. Investing in knowledge society through the European Social Fund.

An initial version of the paper, entitled "Effects of a half-separated (impermeable and permeable) bed on the velocity distribution in open channels" has been presented in the 12th International Conference on Protection and Restoration of the Environment, Skiathos Island, Greece, June 29 to July 3, 2014.

\section{References}

Beavers GS, Joseph DD (1967) Boundary conditions at a naturally permeable wall. J Fluid Mech 30(1):197-207 Bouma TJ, van Duren LA, Temmerman S, Claverie T (2007) Spatial flow and sedimentation patterns within patches of epibenthic structures: combining field, flume and modelling experiments. Cont Shelf Res 27(8): $1020-1045$ 
Chan HC, Leu JM, Lai CJ, Jia Y (2007) Turbulent flow over a channel with fluid-saturated porous bed. J Hydrol Eng 133(6):610-617

Choi CY, Waller PM (1997) Momentum transfer mechanism for water flow over porous media. J Environ Eng ASCE 123:792-799

Ghisalberti M, Nepf H (2009) Shallow flows over a permeable medium: the hydrodynamics of submerged aquatic canopies. Transp Porous Media 78(3):385-402

Huai W, Han J, Zeng Y, An X, Qian Z (2009) Velocity distribution of flow with submerged flexible vegetations based on mixing-length approach. App Math Mech 30(3):343-351

Keramaris E, Pechlivanidis G, Pechlivanidis I, Samaras G (2013) The impact of lateral walls on the velocity profile in an open channel using the PIV method, 13th International Conference on Environmental Sience and Technology (CEST 2013), Athens, Greece

Keramaris E, Pechlivanidis G, Pechlivanidis I (2014) Effects of a half-separated (impermeable and permeable) bed on the velocity distribution in open channels. Proceedings of International Conference Protection and Restoration of the Environment XII, Skiahtos Island, Greece, 2014

Mendoza C, Zhou D (1992) Effects of porous bed on turbulent streamflow above bed. J Hydraul Eng ASCE 111: 1222-1239

Poulikakos D, Kazmierczak M (1987) Forced convection in a duct partially filled with a porous material. J Heat Transf 109:653-662

Raffel M, Willert C, Wereley S, Kompenhans J (2007) Particle image velocimetry: a practical guide. SpringerVerlag

Sahraoui M, Kaviany M (1992) Slip and no-slip velocity boundary conditions at interface of porous, plain media. Int J Heat Mass Transf 35:927-943

Vafai K, Thiyagaraja R (1987) Analysis of flow and heat transfer at the interface region of a porous medium. Int J Heat Mass Transf 30(7):1391-1405

Velasco D, Bateman A, Redondo JM, Demedina V (2003) An open channel experimental and theoretical study of resistance and turbulent characterization over flexible vegetated linings. Flow Turbul Combust 70:69-88

Wereley ST, Meinhart CD (2010) Recent advantages in micro-particle image velocimetry. Annu Rev Fluid Mech 42(1):557-576

Wilson CAME, Stoesser T, Bates PD, Batemann Pinzen A (2003) Open channel flow through different forms of submerged flexible vegetation. J Hydrol Eng 129(11):847-853

Zhu C, Hao W, Chang X (2014) Vertical velocity distribution in open-channel flow with rigid vegetation. Sci World J, Volume 2014

Keramaris E, Pechlivanilis G (2015) The influence of transition from vegetation to gravel bed and vice versa in open channels using the PIV method. Water Util J 10:35-41 